\title{
Optimized electro- and wet-spinning techniques for the production of polymeric fibrous scaffolds loaded with bisphosphonate and hydroxyapatite
}

\author{
Dario Puppi ${ }^{1}$, Anna Maria Piras ${ }^{1}$, Federica Chiellini $^{1}$, Emo Chiellini ${ }^{1 *}$, Albino Martins ${ }^{2,3}$, \\ Isabel B. Leonor ${ }^{2,3}$, Nuno Neves ${ }^{2,3}$ and Rui Reis ${ }^{2,3}$ \\ ${ }^{1}$ Laboratory of Bioactive Polymeric Materials for Biomedical and Environmental Applications (BIOlab), Department of Chemistry and \\ Industrial Chemistry, University of Pisa, Italy \\ ${ }^{2} 3$ B's Research Group - Biomaterials, Biodegradables and Biomimetics, Department of Polymer Engineering, University of Minho, \\ Headquarters of the European Institute of Excellence on Tissue Engineering and Regenerative Medicine, Guimarães, Portugal \\ ${ }^{3}$ IBB - Institute for Biotechnology and Bioengineering, PT Government Associated Laboratory, Guimarães, Portugal
}

\begin{abstract}
This research activity was aimed at the development of composite bioactive scaffolds made of biodegradable three-arm branched-star poly ( $\varepsilon$-caprolactone) ( $\left.{ }^{*} \mathrm{PCL}\right)$, hydroxyapatite nanoparticles (HNPs) and clodronate (CD), a bisphosphonate that has demonstrated efficacy in the treatment of various bone diseases and as an anti-inflammatory drug. During the experimental work, the processing conditions for the fabrication of fibrous meshes, by either electrospinning or wetspinning, were optimized. Stemming from a previous research activity on electrospinning of *PCL, *PCL/HNPs 3D meshes were developed, evaluating the influence of fabrication parameters on the fibres' morphology. By exploiting the binding affinity of bisphosphonates for hydroxyapatite, a methodology was set up for obtaining a physical linkage between CD and HNPs, with the aim of having a dual bioactive system loaded into *PCL fibrous mats. Fibres loaded with either CD or CD-HNP particles were thus produced and analysed by scanning electron microscopy for their morphology and by energy dispersive X-ray spectroscopy for their elemental composition. Copyright (c) 2010 John Wiley \& Sons, Ltd.
\end{abstract}

Received 5 March 2010; Accepted 15 April 2010

Keywords electrospinning; wet-spinning; star polymers; bisphosphonates; scaffolds; tissue engineering

\section{Introduction}

Bisphosphonates (BPs) are a class of bioactive agents inhibiting bone resorption and ectopic calcification, widely used in the therapy of diseases caused by altered metabolism of bone tissue, such as Paget's disease, tumour-associated osteolysis and hypercalcaemia, osteoporosis and primary hyperparathyrosis (Ezra and Golomb, 2000; Rogers et al., 2000; Martin and Grill, 2000).

\footnotetext{
*Correspondence to: Emo Chiellini, Laboratory of Bioactive Polymeric Materials for Biomedical and Environmental Applications (BIOlab), via Vecchia Livornese 1291, 56010 San Piero a Grado (Pi), Department of Chemistry and Industrial Chemistry, University of Pisa, Italy.

E-mail: emochie@dcci.unipi.it
}

They possess a chemical structure similar to that of pyrophosphate, an endogenous regulator of bone mineralization produced by many anabolic processes, with the advantage of a higher resistance to hydrolysis and chemical stability. BP effects are due to their incorporation in the mineral part of bone, enabling interaction with osteoclasts and osteoblasts. Thanks to their high affinity for divalent metal ions, BPs bind strongly to the calcium phosphate crystals of bone hydroxyapatite (HA), inhibiting their growth, aggregation and dissolution (Fleisch et al., 1969, 1970; Francis et al., 1969; Meyer and Nancollas, 1973; Hansen et al., 1976). Moreover, depending on their molecular structure, BPs can inhibit bone resorption through various biochemical pathways with subsequent different effects, such as inhibition of osteoclast formation, osteoclast apoptosis 
or/and by affecting the metabolic activities of osteoblasts (Rogers et al., 2000; Van Beek et al., 2002; Horie et al., 2003).

The major disadvantages associated with systemic use of the clinically employed BPs are poor absorption and adverse gastrointestinal effects in oral administration therapy as well as local tissue damage and irritation at the injection site (Ezra and Golomb, 2000). A localized and controlled BP delivery system can allow for improved bioavailability and safety. It has been reported that a topical treatment allows for overcoming limitations associated to systemic use, with the advantages of having a controlled therapeutic dosage in the region of interest and BP distribution to avascular areas in necrotic bone tissue (Astrand and Aspenberg, 2004). Implanted BP-releasing systems can be employed for the optimization of hypercalcaemia treatment in tumourinduced osteolysis (Ezra and Golomb, 2000) and to improve the local effect in confined dental bone surgery (Nafea et al., 2008). Furthermore, providing implantable bioabsorbable systems with BP-releasing features can prevent osteolytic lesions caused by the inflammatory processes induced by polymer degradation products (Huolman and Ashammakhi, 2007).

Various BPs releasing systems for bone applications have been developed such as coatings for titanium or stainless-steel screws (Tengvall et al., 2004), BPcomplexed HA implants (Denissen et al., 1997, 2000), BPs combined to frozen cancellous bone allografts (Jeppsson et al., 2003) and BP-releasing poly(lactic-co-glycolic) rods for bone fixation (Huolman and Ashammakhi, 2007). The possibility of BP encapsulation into microparticles made of chitosan (Samdancioglu et al., 2006; Patashnk et al., 2009) or poly(lactic-co-glycolic acid) (Perugini et al., 2001; Weidenauer et al., 2003; Nafea et al., 2008) has been also reported.

Poly ( $\varepsilon$-caprolactone) (PCL) is a semicristalline polymer that has been widely proposed for bone regeneration application because of its biocompatibility and degradation properties (Williams et al., 2005; Koh et al., 2006; Rai et al., 2007; Puppi et al., 2010a). In biological conditions, it can be degraded by microorganisms as well as by hydrolytic mechanisms and its slow degradation rate can be tuned to match with bone tissue ingrowth (Hutmacher, 2000; Savarino et al., 2007). However, the implantation of polymer substitutes into bone defects typically presents various drawbacks, such as lack of immediate adhesion to the surrounding tissue because of an inflammatory reaction, encapsulation into fibrous tissue and mechanical strength reduction associated with material degradation (Taylor et al., 1994; Kokubo et al., 2003; Schiller and Epple, 2003). The incorporation of HA particles into polymer matrices has been investigated as an effective means of improving the osteoconductivity and mechanical properties of bone implants and create a $\mathrm{pH}$ buffer against the acidic degradation products of the polymeric matrices (Ural et al., 2000; Kikuchi et al., 2004; Kim et al., 2004; Koh et al., 2006; Puppi et al., 2010a).
This research activity was aimed at the development of new BP-releasing bioabsorbable scaffolds for bone tissue-engineering applications. Clodronic acid disodium salt (clodronate, CD), an efficient anti-osteolytic agent with approved clinical record and low rate of adverse events (Selander et al., 1996; Fleisch, 2000; Huolman and Ashammakhi, 2007), was chosen in this study as a BP model. CD has also demonstrated efficacy as anti-inflammatory agent and in stimulating osteoblast differentiation in osteoblast-like and pluripotent mesenchymal cell culture (Horie et al., 2003; Itoh et al., 2003). The three-dimensional (3D) carriers developed within this experimental project are polymeric and composite fibrous meshes produced by either electrospinning or wet-spinning techniques. Since fibre dimension and mesh porosity can greatly affect drug release kinetics, our aim was to develop BP-loaded polymeric meshes with a different structural scale and texture by employing the two techniques mentioned. A three-arm branched star poly ( $\varepsilon$ caprolactone) (*PCL) (Balakrishnan et al., 2004, 2006), a biocompatible polymer which has been already investigated for the production of electrospun fibrous meshes for tissue engineering applications (Puppi et al., 2010b), was employed. The processing conditions for the production of *PCL meshes loaded with either HA nanoparticles (HNPs) or CD were optimized and correlated to mesh morphology. Exploiting the binding affinity of BPs for HA (Rogers et al., 2000; Leu et al., 2006), a methodology for obtaining a physical linkage between CD and HNPs was investigated. The incorporation of particles of such complex into *PCL fibres was also studied in order to introduce a further control over drug release as well as to increase the osteoconductivity of polymer meshes. The meshes produced were characterized for their morphology by scanning electron microscopy (SEM) and for composition by energy dispersive X-ray spectroscopy (EDS).

\section{Materials and methods}

\subsection{Materials}

Three-arm branched star poly( $\varepsilon$-caprolactone) (*PCL, MW 189000 Da) was kindly supplied by Michigan Biotechnology Institute; HA nanoparticles (HNPs, size $<200 \mathrm{~nm}$ ) were bought from Sigma-Aldrich and clodronic acid disodium (CD) was kindly supplied by Abiogen Pharma-Pisa (Italy). All the solvents and chemical reagents were purchased from Sigma-Aldrich (Italy) and used as received.

\subsection{Preparation of CD-HNP complex and study of loading efficiency}

$500 \mathrm{mg}$ CD was dissolved in $10 \mathrm{ml}$ distilled water at room temperature with stirring for $2 \mathrm{~h}$. Then, $250 \mathrm{mg}$ HNPs powder were added to the CD solution and the mixture was incubated under gentle stirring for $1 \mathrm{~h}$ at 
room temperature. CD binding efficiency was evaluated by measuring the free $\mathrm{CD}$ in the supernatant. Free CD was complexed with copper ions $\left(\mathrm{Cu}^{2+}\right)$ in nitric acid solution, and its concentration was determined by ultraviolet spectrophotometry (UV) at $236 \mathrm{~nm}$ (standard curve, 0.025-1.0 mM; $\left.R^{2}=0.993\right)$ as described by Ostović et al. (1993). The binding efficiency $(\eta)$ of the physical reaction between CD and HNPs was calculated as follows:

$$
\eta=\left(C D_{\mathrm{i}}-C D_{\mathrm{f}}\right) / C D_{\mathrm{i}}
$$

where $C D_{\mathrm{i}}$ is the input drug and $C D_{\mathrm{f}}$ is the free drug in the supernatant.

\subsection{Development of fibrous meshes by either electrospinning or wet-spinning}

Polymer and composite fibrous meshes were produced by processing plain *PCL solutions or suspensions of HNPs, CD or CD-HNP particles in *PCL solution, respectively. The processing conditions for the production of fibres by either electrospinning or wet-spinning were investigated. The prepared meshes were analysed for their morphology and composition by SEM and EDS, respectively.

\subsubsection{Preparation of polymer solutions and polymer/CD/HNP mixtures}

${ }^{*}$ PCL was dissolved in acetone at $40^{\circ} \mathrm{C}$, under gentle stirring for $2 \mathrm{~h}$ to obtain homogeneous solutions of various concentrations. For the production of composite fibres, $\mathrm{CD}, \mathrm{HNPs}$ or CD-HNP particles were added to the polymer solution and left under vigorous stirring for $2 \mathrm{~h}$ to achieve their homogeneous dispersion.

On the base of some preliminary investigations, the weight ratio between HNPs and *PCL in solutions for the production of fibres loaded with either HNPs or CD-HNP particles, was chosen to be $25 \%$ by weight. Therefore, considering the binding efficiency between CD and HNPs, the theoretical weight ratio between $\mathrm{CD}$ and ${ }^{*} \mathrm{PCL}$ in the solutions for the production of fibres loaded with CD-HNPs was 0.075. This value was employed also for the fabrication of CD-loaded *PCL fibres.

\subsubsection{Production of electrospun fibre meshes}

A $15 \% \mathrm{w} / \mathrm{v} *$ PCL solution was placed in a syringe equipped with a metallic needle, with an inner diameter (i.d.) of $0.84 \mathrm{~mm}$, and the solution feeding rate was controlled by a syringe pump (KDS100, KD Scientific, MA). Using a semi-commercial high-voltage power supply, an electric field was applied between the metallic needle of the syringe and an electrical grounded aluminium collector. The applied voltage was $25 \mathrm{kV}$ and the distance from the needle tip to the collector $15 \mathrm{~cm}$. The variation of the processing conditions for the production of *PCL meshes was evaluated in order to optimize the fibres mesh morphology. In particular, the influence of solution feed rate was investigated in the range $1-16 \mathrm{ml} / \mathrm{h}$. The electrospun mats were removed, placed in a vacuum chamber for at least $48 \mathrm{~h}$ to allow the evaporation of the residual organic solvent and then stored in a desiccator.

\subsubsection{Production of $3^{D}$ wet-spun fibre meshes}

A polymer solution was loaded into a plastic syringe (5 ml) with a metallic needle $(0.5 \mathrm{~mm}$ i.d.) attached to it. The syringe was connected to a programmable syringe pump (KD Scientific, World Precision Instruments, UK) to inject the polymer solution at controlled pumping rate to allow the formation of non-woven web directly into the coagulation bath. The non-woven web was formed during the processing by the random movement of the coagulation bath. Various processing parameters, such as solvent, polymer concentration, coagulation bath and solution flow rate, were varied to optimize fibre morphology. Either acetone or chloroform was employed as solvent and methanol, ethanol and water were tested for the coagulation bath. Spun meshes were left in the coagulation bath for $24 \mathrm{~h}$, then removed, placed in a vacuum chamber for $48 \mathrm{~h}$ and finally stored in a desiccator.

\subsection{Scanning electron microscope (SEM) and energy dispersive X-ray spectroscopy (EDS) analysis}

Samples were gold-coated using a sputter-coater set at $15 \mathrm{~mA}$ for $20 \mathrm{~min}$ and mesh morphology was investigated by using SEM instruments (Leica 360; FEI Nova 200). Mesh elemental composition was investigated by EDS analysis (EDAX Pegasus X4M), based on the energy and intensity distribution of X-ray signals generated by the electron beam striking the surface of the specimen. Three random areas $(300 \times 400 \mu \mathrm{m})$ of each sample were analysed for their elemental composition.

\section{Results and discussion}

\subsection{Development of CD-HNPs complex}

The high affinity of BPs for HA, due to their ability to form a 3D structure with divalent metal ions (Rogers et al., 2000; Leu et al., 2006), is the basis of their bonetargeting property. A few recent experimental studies have proposed HA as the carrier for the controlled delivery of BPs adopting different strategies, such as incorporation of BPs into HA crystals (McLeod et al., 2006; Boanini et al., 2007) or BP-HA physical binding (Seshima et al., 2006; Ong et al., 2008).

In the present study, we investigated a straightforward simple method for obtaining a physical binding between CD and HNPs with $\eta$ (yield) of around 15\%. Figure 1 shows micrographs of HNPs and CD powders as well as that of CD-HNPs powder, recovered after centrifugation 

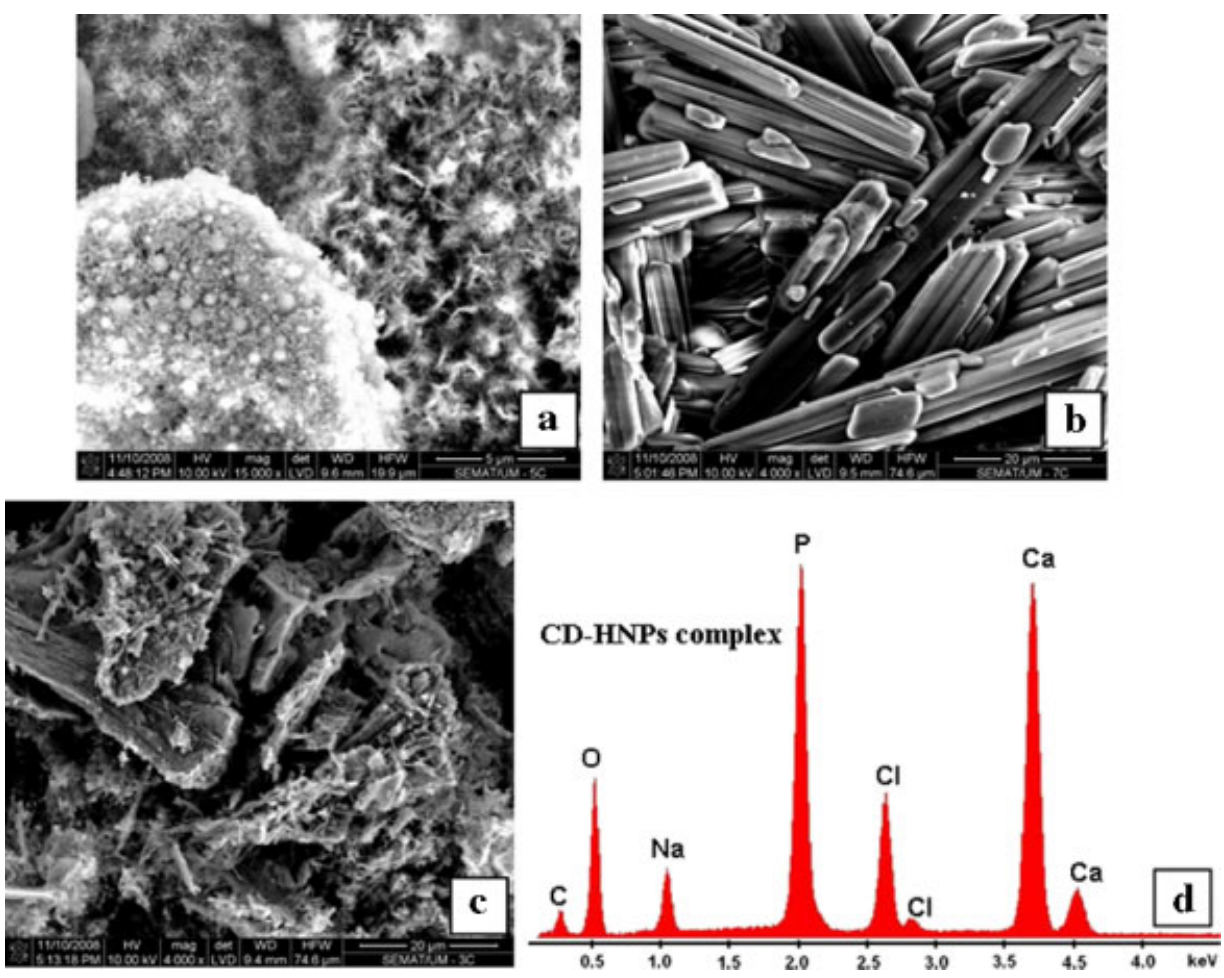

Figure 1. SEM micrographs: (a) HNPs powder; (b) CD powder; (c) CD-HNPs powder. (d) Representative EDS spectrum of CD-HNPs complex

of the reaction mixture. Compared to the native CD and HNP morphology, in the micrograph of the recovered CD-HNPs a coating of CD structures with HNPs can be detected, thus indicating the formation of the complex CD-HNPs at least in the dry powder state (Figure 1c).

EDS analysis of the recovered CD-HNPs powders showed the presence of chlorine $(\mathrm{Cl})$ and sodium $(\mathrm{Na})$, correlated to the presence of $\mathrm{CD}$, and calcium (Ca), associated to HNPs (Figure 1d). Moreover, from comparison among spectra of different randomly taken fields, it appears that CD was uniformly distributed along powder specimens (data not shown).

\subsection{Development of *PCL-based electrospun meshes}

In the last 10 years, electrospinning has been gaining a widespread interest for applications in drug delivery and tissue engineering. Electrospinning enables great flexibility in materials selection, drug-loading methods and choice of the appropriate loading agent. These, together with various inherent features of electrospun meshes (such as high surface area : volume ratio and high and interconnected porosity), can enhance drug loading and mass transfer properties, offering a broad spectrum of advanced design solutions for the fine control of drug release kinetics (Ashammakhi et al., 2007; Detta et al., 2010). In the last years a few studies have investigated the possibility of incorporating therapeutic and bioactive agents into electrospun fibres without affecting mesh morphology and the bioactivity of loaded agents (Cui et al., 2006; Piras et al., 2006; Xie and Wang, 2006; Xu et al., 2006; Zheng-Ming et al., 2006; Nie and Wang, 2007; Maretschek et al., 2008; Piras et al., 2008; Puppi et al., 2009). Moreover, electrospun mats composed of randomly orientated fibres with diameters down to the nanometer scale are suitable for replicating the physical structure of the extracellular matrix of biological tissues, and they have been shown to influence in vitro cell adhesion, proliferation and phenotypic behaviour (Bhattarai et al., 2004; Shin et al., 2004; Fujihara et al., 2005; Badami et al., 2006; Stitzel et al., 2006; Martins et al., 2007, 2009a, 2009b; Araujo et al., 2008; da Silva et al., 2008; Pinho et al., 2009).

During the present research activity, we investigated the processing of suspensions of HNPs, CD or CD-HNPs in *PCL solution by applying the electrospinning conditions optimized in a previous related study on *PCL electrospun fibres $(15 \% \mathrm{w} / \mathrm{v}$ polymer concentration, $\mathrm{V}=25 \mathrm{kV}$ and $\mathrm{d}=15 \mathrm{~cm}$ ) (Puppi et al., 2010b) and by studying the influence of solution feed rate on mesh morphology.

\subsection{1. *PCL/HNPs electrospun meshes}

In the recent years, a few published works have reported the inclusion of HA particles into electrospun polymer fibres as means for improving mesh osteoconductivity and mechanical properties (Ito et al., 2005; Hae-Won et al., 2006; Catledge et al., 2007; Dai and Shivkumar, 2007).

As shown in Figures 2a-c, the addition of HNPs to the electrospinning solution led to the formation of fibre morphology defects. By increasing the feed rate, the 

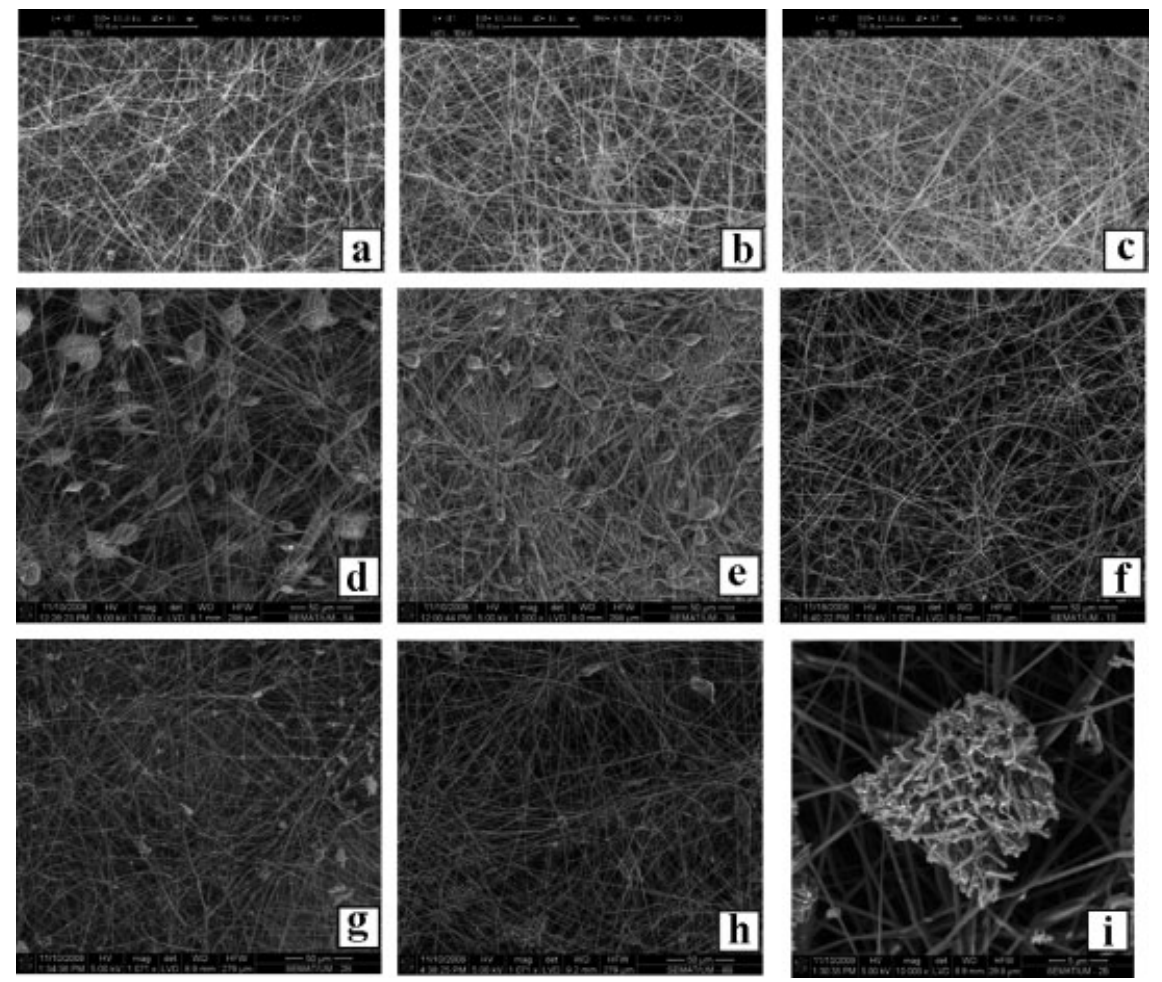

Figure 2. SEM micrographs of *PCL-based electrospun samples $\left(\mathrm{C}_{* \mathrm{PCL}}=15 \% \mathrm{w} / \mathrm{v}, \mathrm{V}=25 \mathrm{kV}\right) .{ }^{*} \mathrm{PCL} / \mathrm{HNPs}$ composite meshes: (a) $\mathrm{F}$ $=16 \mathrm{ml} / \mathrm{h}$; (b) F= $8 \mathrm{ml} / \mathrm{h}$; (c) F= $4 \mathrm{ml} / \mathrm{h}$. CD-loaded *PCL meshes: (d) $\mathrm{F}=16 \mathrm{ml} / \mathrm{h}$; (e) F= $8 \mathrm{ml} / \mathrm{h} ;(\mathrm{f}) \mathrm{F}=2 \mathrm{ml} / \mathrm{h}$. ${ }^{*}$ PCL meshes loaded with CD-HNP particles: (g) $\mathrm{F}=16 \mathrm{ml} / \mathrm{h}$; (h) $\mathrm{F}=4 \mathrm{ml} / \mathrm{h}$; (i) $\mathrm{F}=16 \mathrm{ml} / \mathrm{h}$, high magnification

formation of defects in the analysed meshes was inhibited. EDS analysis confirmed the presence (Figure 3a) and the homogeneous distribution of phosphorus (P) and $\mathrm{Ca}$ correlated to HNPs loading.

\subsection{2. $C D$-loaded *PCL electrospun meshes}

SEM analysis of electrospun CD-loaded *PCL meshes showed a fibrous structure characterized by fibre defects, in the form of beads along the fibres (Figures $2 \mathrm{~d}-\mathrm{f}$ ). Both the number per unit area and the size of beads were gradually decreased by decreasing the solution feed rate from $16 \mathrm{ml} / \mathrm{h}$ to $1 \mathrm{ml} / \mathrm{h}$. EDS analysis was performed to investigate whether heterogeneous drug distribution was responsible for mesh defects and to analyse the beads' composition. Spectra of CD-loaded meshes displayed low-energy peaks corresponding to the elements of $\mathrm{CD}$ (Figure 3b). This can be related to the low amount of CD added to the polymer solution and/or to the distribution of the drug in the fibre section. Moreover, there were no remarkable differences between the composition of fibres and beads, indicating that the formation of such defects was due to the electrospinning setting, which initially was not optimized, and not to a heterogeneous drug distribution into the mesh microstructure.

\subsection{3. *PCL meshes loaded with CD-HNP particles}

Fibrous meshes loaded with CD-HNP particles were produced with no remarkable influence of the feeding rate on fibre morphology (Figure 2g, h). Figure 3c shows a representative EDS spectrum of this kind of mesh; comparing the EDS spectra taken from different areas in the same sample, the elements composing both $\mathrm{CD}$ $(\mathrm{P}, \mathrm{Cl}, \mathrm{Na})$ and HNPs (P, Ca) were uniformly distributed in the analysed meshes. However, some microaggregates (Figure 2i) with morphology similar to that observed in CD-HNPs powder (Figure 1c) were observed in highmagnification micrographs. EDS analysis revealed that these microaggregates were mainly composed of the elements of $\mathrm{CD}$ and HNPs (Figure 3d). The presence of such microaggregates, mainly composed of CD-HNP particles not incorporated into *PCL fibres, may influence the release kinetics of the loaded meshes, contributing to a possible initial burst release.

\subsection{Development of wet-spun meshes}

Wet-spinning is a non-solvent-induced phase inversion technique allowing for polymer fibre production through an immersion-precipitation process. In detail, a continuous polymer fibre is produced by precipitation of a polymer solution filament in a coagulation bath composed of a poor solvent (non-solvent) or a non-solvent-solvent mixture with respect to the processed polymer. An homogeneous solution filament, composed of polymer, solvent and possible additives, solidifies because of polymer desolvation, caused by solvent-non-solvent exchange.

A number of studies have reported the development of wet-spun fibres made of natural polymers, such as 

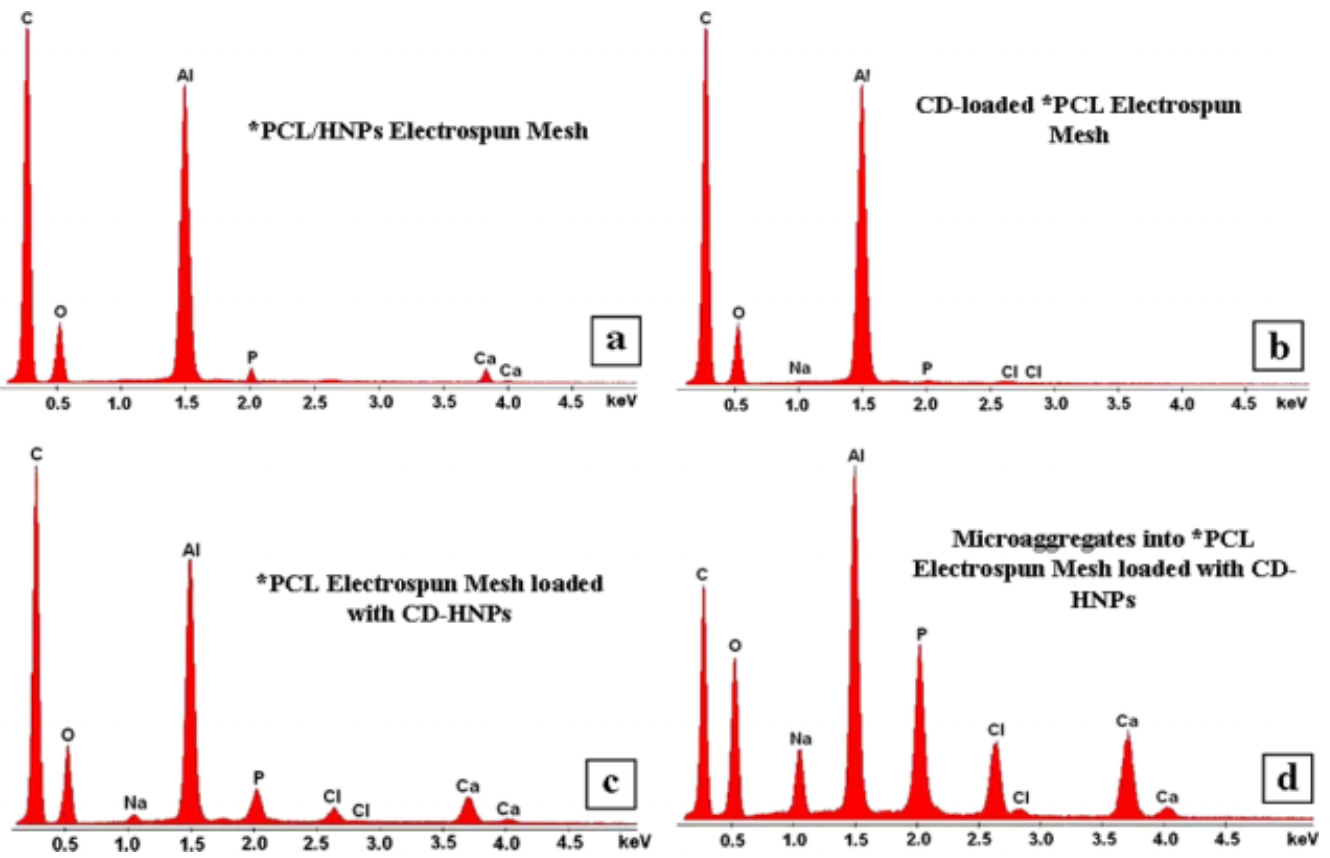

Figure 3. Representative EDS spectra of electrospinning samples: (a) *PCL/HNPs composite mesh; (b) CD-loaded *PCL mesh; (c) *PCL mesh loaded with CD-HNPs; (d) microaggregate into *PCL mesh loaded with CD-HNPs
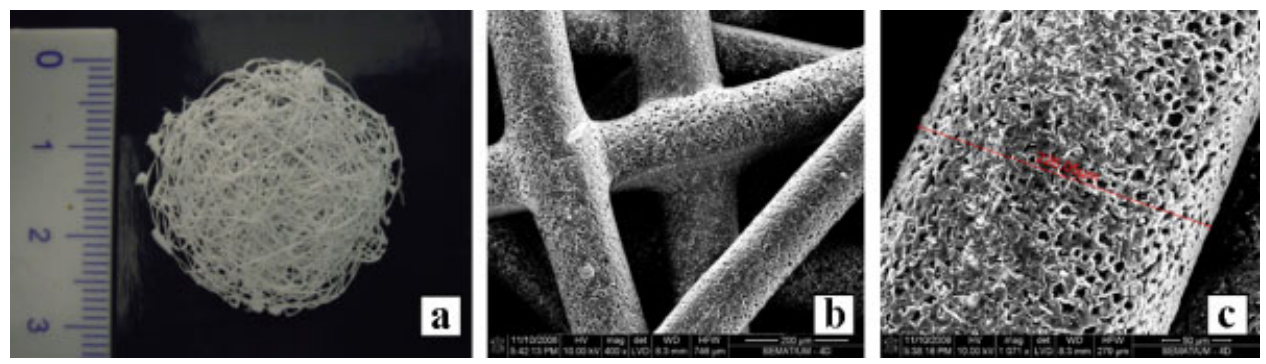

Figure 4. Wet spun *PCL ( F = $1.75 \mathrm{ml} / \mathrm{h})$ : (a) photograph of 3D mesh; (b) SEM micrograph of mesh; (c) SEM micrograph of single fibre

chitosan (Tuzlakoglu et al., 2004; Tuzlakoglu and Reis, 2008) and regenerated silk fibroin (Yao et al., 2001; Um et al., 2004; Lee et al., 2007). In recent years, some research has proposed wet-spun fibres as carriers for drug release, such as either chitosan (Denkbas et al., 2000) or PLLA fibres (Gao et al., 2007) loaded with 5fluorouracil. This technique was also proposed to develop scaffolds for tissue-engineering applications, based in PCL fibres (Williamson and Coombes, 2004), braided PLLA or chitosan fibres (Zhang et al., 2007) and starch-based fibres (Tuzlakoglu et al., 2009).

During this experimental activity, the wet-spinning process for obtaining the *PCL-based wet-spun fibres loaded with HNPs, CD or CD-HNPs were investigated by improving the processing conditions, such as the solvent-non-solvent system, polymer concentration and solution feed rate.

\subsection{1. *PCL wet-spun meshes}

The production of fibrous meshes by wet-spinning was tested with *PCL solutions and two different solvents (acetone and chloroform), as well as various polymer concentrations (10-20\% w/v) and three different nonsolvents (methanol, ethanol, water), to optimize the processing conditions. When chloroform was employed as solvent, proper polymer coagulation was not achieved for any of the above-mentioned non-solvents. Employing acetone as solvent and ethanol as non-solvent, phase inversion took place, allowing for the coagulation of the spun solution in the form of a polymer fibre. In this case, the polymer concentration giving by the best fibre morphology was found to be $15 \% \mathrm{w} / \mathrm{v}$. The influence of solution flow rate on fibre morphology was further studied in the range $1.5-3.5 \mathrm{ml} / \mathrm{h}$. The results showed that the fibres' morphology was improved when using the flow rate of $1.75 \mathrm{ml} / \mathrm{h}$ (Figure $4 \mathrm{a}$ ). This condition produced a fibre diameter in the range $100-250 \mu \mathrm{m}$ and a porous fibre structure with a pore size of few micrometers (Figure 4b, c). Moreover, the fibres looked fused at fibre-fibre contact points, forming a cohesive non-woven web.

Upon immersion of a polymer solution into a coagulation bath, the non-solvent diffuses into the 

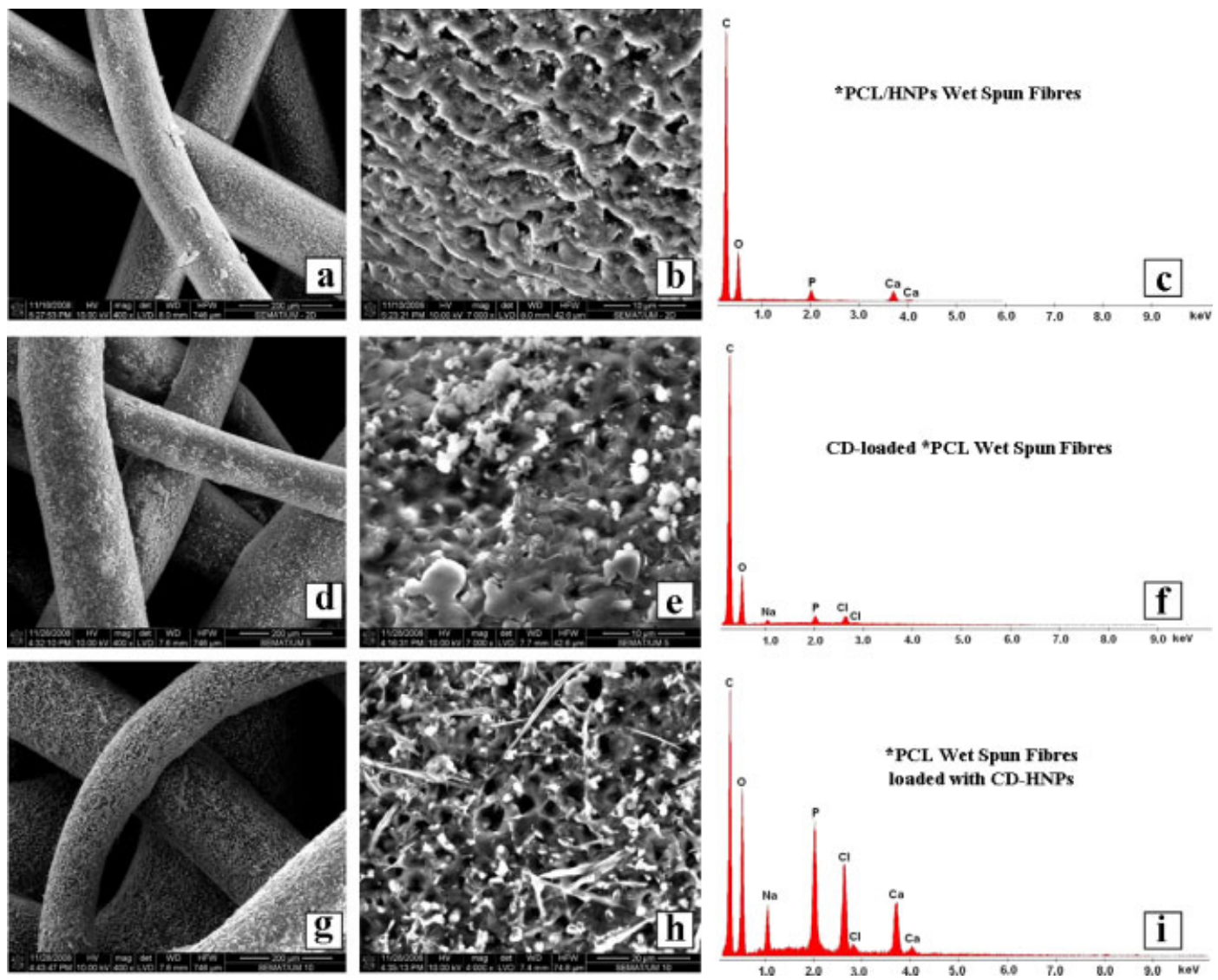

Figure 5. Wet spun *PCL-based meshes: (a) SEM micrograph of *PCL/HNPs mesh; (b) SEM micrograph of fibre surface of *PCL/HNPs mesh; (c) EDS spectrum of *PCL/HNPs fibre; (d) SEM micrograph of CD-loaded *PCL mesh; (e) SEM micrograph of fibre surface of CD-loaded *PCL mesh; (f) EDS spectrum of CD-loaded *PCL fibre; (g) SEM micrograph of *PCL mesh loaded with CD-HNP particles; (h) SEM micrograph of fibre surface of *PCL mesh loaded with CD-HNP particles; (i) EDS spectrum of *PCL fibre loaded with CD-HNPs

polymer solution, whereas the solvent diffuses into the bath. Solvent-non-solvent counter-diffusion lowers the polymer solubility and induces a phase separation into a polymer-rich phase and a polymer-lean phase. A dense polymer matrix surrounding the dispersed polymer-lean droplets, which will form the pores, is consequently formed. Usually a dense, non-porous layer, due to the instantaneous non-solvent diffusion into the polymer solution, is observed at the interface with the coagulant (Tsay and Mchugh, 1990, 1992; Wienk et al., 1996). As reported in the literature, the formation of a spongy morphology with a porous surface interfacing with the coagulating fluid is due to delayed liquid-liquid demixing (Wienk et al., 1996; Barton et al., 1997).

What is observed in the SEM micrographs of wet-spun *PCL fibres, concerning both the porous morphology of the fibre outer surface and the bonding at the fibre-fibre contact points, indicates that in our case the inversion phase process was governed by delayed precipitation and that the filament was not completely solidified when it settled at the bottom of the bath container.

On the basis of this preliminary investigation, the optimized processing conditions were $15 \% \mathrm{w} / \mathrm{v}$ polymer concentration, acetone as solvent, ethanol as non-solvent and $1.75 \mathrm{ml} / \mathrm{h}$ flow rate. The composition of the starting suspensions selected for the production of *PCL fibres loaded with HNPs, CD or CD-HNP particles was the same as that already employed for the electrospinning technique.

\subsection{2. ${ }^{*} P C L / H N P s$ wet-spun meshes}

3D fibrous meshes of *PCL/HNPs composite fibres were fabricated by the coagulation of a filament of HNPs suspension in the polymer solution. SEM analysis from *PCL/HNPs meshes revealed a more narrow fibre size distribution, comparing to bare polymer matrices, with fibre diameters in the range $100-200 \mu \mathrm{m}$. In addition, *PCL/HNPs meshes showed a less porous surface and no fusion between fibres at the crossing points (Figure 5a, b).

It is known that the additives loaded into polymer solution may have an effect on the final morphology of the polymeric porous systems produced by phase inversion. Their presence is mainly reflected on both process thermodynamics and kinetics (Strathmann et al., 1975; Reuvers and Smolders, 1987; Mulder, 1996; Kim and Lee, 1998).

In this case, a possible effect of the addition of HNPs to polymer solution could be a faster solidification of the filament, generating a less porous fibre morphology. 
In a speculative manner, this phenomenon may be attributed to a pre-existing interaction between polymer and additive, which indeed has an effect on polymer solvation and consequently on the coagulation process.

Figure $5 c$ shows a representative EDS spectrum of HNPs-loaded *PCL fibres. Chemical composition analysis of fibre surface revealed the presence and homogeneous distribution of $\mathrm{P}$ and $\mathrm{Ca}$ elements, unequivocally associated with the presence of HNPs.

\subsubsection{CD-loaded ${ }^{*} P C L$ wet-spun meshes}

The possibility of producing wet-spun fibres loaded with bioactive agents has been shown in a few studies over recent years (Denkbas et al., 2000; Gao et al., 2007; Chang et al., 2008). In the present study, 3D CD-loaded fibre meshes were successfully produced by coagulation of a filament of $\mathrm{CD} /{ }^{*} \mathrm{PCL}$ suspension (Figure $5 \mathrm{~d}$, e). The addition of $\mathrm{CD}$ had a remarkable influence on mesh morphology: the fibre dimensions changed to a wider diameter range $(150-350 \mu \mathrm{m})$ and no fusion was observed between fibres at the crossing points. The reduced surface porosity and the absence of fibre-fibre fusion suggest that a faster liquid-liquid demixing takes place in comparison to the processing of bare polymer fibres.

EDS analysis (Figure 5f) confirmed the presence and the homogeneous distribution of CD diagnostic atomic elements $(\mathrm{Na}, \mathrm{P}$ and $\mathrm{Cl})$. In addition, some white spots were observed on the fibre surfaces and they were analysed for their elemental composition to determine whether they were mainly composed of elements of CD. The small differences in intensity of analogue energy peaks in the spectra of the wide fibre surface areas and the white spots revealed that there was no significant drug segregation onto the fibre surface.

\subsubsection{Wet-spun fibres loaded with CD-HNP particles}

By processing a suspension of CD-HNP particles in * PCL solution, composite meshes with fibre diameters in the range $100-200 \mu \mathrm{m}$ were produced. As shown by SEM analysis (Figure $5 \mathrm{~g}, \mathrm{~h}$ ), the fibre surface was characterized by a porous morphology and appeared to be covered with aggregates of CD-HNP particles. This was confirmed by EDS analysis of the fibre surface, showing high-energy peaks associated with elements composing CD and HNPs (Na, Cl, P and Ca) (Figure 5i). Similarly to what was observed for fibres loaded with either HNPs or CD, the surface porosity was reduced in comparison with unloaded fibres and the fibres did not show fusion at the crossing points.

\subsection{General remarks on the results achieved}

This section aims to provide a generalized discussion of the techniques utilized for the production of $3 \mathrm{D}$ polymeric scaffolds.
By applying the two different techniques investigated, we obtained 3D meshes composed of *PCL fibres with different texture scales and loaded with bioactive agents for potential application in bone repair. In both cases, the addition of loading agents (HNPs, CD or CD-HNPs) to the polymer solutions affected the morphology of the final fibrous structure. In detail, the addition of either HNPs or $\mathrm{CD}$ led to the formation of defects along the electrospun fibres, which could be minimized by varying the solution feed rate and affected the wet-spinning solidification process, causing changes in mesh morphology in terms of fibre-fibre contact point, fibre diameter and fibre porosity. In the case of meshes produced from suspensions of CD-HNP particles, aggregates mainly composed of CD and HNPs elements, which were not incorporated into polymer fibres, were observed in both electrospun and wet-spun samples. The inclusion of CD-HNP particles into fibres may present advantages over the loading of $\mathrm{CD}$ alone, such as an increase in osteoconductivity, due to the presence of the inorganic phase, and the introduction of a further control over CD release kinetics because of the physical binding between CD and HA. However, the presence of such microaggregates should be taken into consideration when studying drug release kinetics, because it may influence the behaviour of the loaded meshes, contributing to a possible initial burst in the release profile. In addition, such aggregates may cause great loss of the drug and the inorganic phase due to their solubilization or leaching during the pre-treatment stages before in vivo implantation (e.g. sterilization, in vitro scaffold preconditioning) causing a decrease of the actual loading of the implant.

During this study, it was observed that the developed electrospun fibres have a diameter of a few micrometers, while wet-spun fibres have a diameter of a few hundred micrometers. As the diameter of polymeric fibres shrinks, a much larger specific surface area can result, and the ratio of the polymer chains and of possible functional groups exposed to the surrounding environment can proportionally increases. As a consequence, more binding sites to cell membrane receptors are available, favouring cell attachment and proliferation, and mass transport phenomena throughout the fibrous construct are enhanced, improving cell nutrients and waste exchange (Zhang et al., 2005; Detta et al., 2010). In addition, such influence on the mechanism and/or kinetics of the transport processes in the porous system will likely increase the material degradation rate and the release kinetics of loaded bioactive factors. However, it should be taken into account that the non-woven ultra-fine fibre structure typical of electrospun meshes presents pores in the nanometer scale, or in any case with the pore dimensions not larger than few micrometers. It is possible that this factor limits cell migration through the mesh, such that nanofibre constructs have been also proposed as an effective means to prevent post-surgery abdominal adhesion by providing a barrier function (Sill and von Recum, 2008). In addition, electrospinning enables polymeric meshes with 
a thickness not larger than few millimeters to be obtained, while wet-spun constructs can have a thickness of some centimeters. In order to combine the advantages of each kind of fibre and minimize the aforementioned concerns, it would be interesting to combine the electrospun and wet-spun fibres developed during this study into a multi-scale network structure. Previous studies on such tissue-engineering scaffolds showed that electrospun ultrafine fibres can enhance the biological performance of the 3D microfibrous structure (obtained by either wet-spinning or rapid prototyping), which provides the required mechanical stability (Tuzlakoglu et al., 2005; Martins et al., 2009a). Moreover, on the hypothesis that the remarkable difference in fibre diameter between electrospun and wet-spun fibres significantly affects mass transfer phenomena, by modulating the ratio between these two kinds of fibres constituting a multi-scale construct it would be possible to vary the release kinetics.

Another aspect to be considered is the need for improvement of the wet-spinning process employed during the present study for the production of 3D structures. Considering that the non-woven morphology was obtained through a continuous random motion of the coagulation tank made by hand, the final fibrous texture depends on the ability of the operator, and the process can be tiring (a scaffold of $5 \mathrm{~mm}$ in thickness is produced in around $30 \mathrm{~min}$, applying the processing conditions optimized in this study). The employment of an automated system for computer control of the tank motion could significantly increase the production efficiency and improve the reproducibility of the mesh morphology. This is an important issue for future research activity.

\section{Conclusions}

Considering the multifunctional biological activity of BPs, we believe that a BP-releasing feature can greatly improve the performance of tissue-engineering scaffolds in inhibiting bone resorption associated with various bone diseases, such as tumour-associated altered bone metabolism or osteoporosis. It would play a main role in preventing osteolytic lesions caused by polymer degradation products, inhibiting post-implantation inflammatory reactions, and in stimulating bone regeneration. This research activity was aimed at the set-up of optimal conditions for the production of CD-loaded biodegradable fibrous scaffolds. In order to have control over the release kinetics of the active agent through the tuning of fibre dimensions and mesh porosity, we have developed fibrous mats with different structural scales and textures, by applying either electrospinning or wet-spinning techniques. ${ }^{*} \mathrm{PCL}$, a novel star branched polymer, was investigated for the production of CD-releasing fibre meshes. Moreover, in order to introduce a further control over drug release based on CD affinity for HA, we developed a methodology for the obtainment of CD-HNP complex particles, achieving 15\% binding affinity. Composite *PCL fibre meshes, obtained by processing suspensions of either HNPs or CD-HNP particles in polymer solution, were successfully produced. The inclusion of the active agents into polymer fibres influenced mesh morphology in the case of both electrospinning and wet-spinning. EDS analysis confirmed the presence of the active agents, loaded into the fibrous meshes. The developed methodologies appear simple and operator-friendly and led to highly reproducible results.

The study is presently ongoing for evaluation of the applicability of the developed meshes as scaffolds for bone repair and tissue engineering. All the prepared scaffolds are currently under characterization for their biodegradation profile, drug release kinetics, mechanical properties, biocompatibility and osteoconductivity.

\section{Acknowledgements}

This work was done within the framework of the European Network of Excellence 'EXPERTISSUES', Project No. NMP3CT-2004-500283. Professor Ramani Narayan of Michigan Biotechnology Institute and Dr Fabio Neggiani of Abiogen Pharma-Pisa are acknowledged for supplying *PCL and CD, respectively.

\section{References}

Araujo JV, Martins A, Leonor IB, et al. 2008; Surface controlled biomimetic coating of polycaprolactone nanofiber meshes to be used as bone extracellular matrix analogues. J Biomater Sci Polym Ed 19: $1261-1278$.

Ashammakhi N, Ndreu A, Piras AM, et al. 2007; Biodegradable nanomats produced by electrospinning: expanding multifunctionality and potential for tissue engineering. J. Nanosci Nanotechnol 7: 862-882.

Astrand J, Aspenberg P. 2004; Topical, single dose bisphosphonate treatment reduced bone resorption in a rat model for prosthetic loosening. $J$ Orthop Res 22: 244-249.
Badami AS, Kreke MR, Thompson MS, et al. 2006; Effect of fiber diameter on spreading, proliferation, and differentiation of osteoblastic cells on electrospun poly(lactic acid) substrates. Biomaterials 27: 596-606.

Balakrishnan S, Krishnan M, Dubois P, et al. 2004; Kinetic and thermodynamic considerations in the synthesis of a new three-arm poly( $\varepsilon$-caprolactone). Polym Eng Sci 44: 1491-1497.

Balakrishnan S, Krishnan M, Narayan R, et al. 2006; Three-arm poly ( $\varepsilon$ caprolactone) by extrusion polymerization. Polym Eng Sci 46: 235-240.

Barton BF, Reeve JL, McHugh AJ. 1997; Observations on the dynamics of nonsolvent-induced phase inversion. $J$ Polym Sci B Polym Phys 35: 569-585.

Bhattarai SR, Bhattarai N, Yi HK, et al. 2004; Novel biodegradable electrospun membrane: scaffold for tissue engineering. Biomaterials 25: 2595-2602.

Boanini E, Gazzano M, Rubini K, et al. 2007; Composite nanocrystals provide new insight on alendronate interaction with hydroxyapatite structure. Adv Mater 19: 2499-2502.

Catledge SA, Clem WC, Shrikishen N, et al. 2007; An electrospun triphasic nanofibrous scaffold for bone tissue engineering. Biomed Mater 2: 142-150.

Chang HI, Lau YC, Yan C, et al. 2008; Controlled release of an antibiotic, 
gentamicin sulphate, from gravity spun polycaprolactone fibers. $J$ Biomed Mater Res A 84A: 230-237.

Cui W, Li X, Zhu X, et al. 2006; Investigation of drug release and matrix degradation of electrospun poly(DL-lactide) fibers with paracetanol inoculation. Biomacromolecules 7: 1623-1629.

da Silva MA, Crawford A, Mundy J, et al. 2008; Evaluation of extracellular matrix formation in polycaprolactone and starchcompounded polycaprolactone nanofiber meshes when seeded with bovine articular chondrocytes. Tissue Eng A 15: 377-385.

Dai X, Shivkumar S. 2007; Electrospinning of hydroxyapatite fibrous mats. Mater Lett 61: 2735-2738.

Denissen H, Martinetti R, van Lingen A, et al. 2000; Normal osteoconduction and repair in and around submerged highly bisphosphonate-complexed hydroxyapatite implants in rat tibiae. $J$ Periodontol 71: $272-278$

Denissen $\mathrm{H}$, van Beek E, van den Bos T, et al. 1997; Degradable bisphosphonate-alkaline phosphatase complexed hydroxyapatite implants in vitro. $J$ Bone Miner Res 12: 290-297.

Denkbas EB, Seyyal M, Piskin E. 2000; Implantable 5-fluorouracil loaded chitosan scaffolds prepared by wet spinning. J Membr Sci 172: 33-38.

Detta N, Puppi D, Errico C, et al. 2010; Polymeric nanofiber constructs in drug delivery and tissue engineering. In NANOPARTICLES: Synthesis, Characterization and Applications, Chaughule RS, Ramanujan RV (eds). American Scientific Publishers: Stevenson Ranch, CA (in press).

Ezra A, Golomb G. 2000; Administration routes and delivery systems of bisphosphonates for the treatment of bone resorption. Adv Drug Del Rev 42: 175-195.

Fleisch H. 2000; Commercially available bisphosphonates. In Bisphosphonates in Bone Disease, 4th edn, Fleisch H (ed). Academic Press: San Diego, CA; 182-206.

Fleisch H, Graham R, Russell G, et al. 1969; Diphosphonates inhibit hydroxyapatite dissolution in vitro and bone resorption in tissue culture and in vivo. Science 165: 1262-1264.

Fleisch HA, Russell RGG, Bisaz S, et al. 1970 The inhibitory effect of phosphonates on the formation of calcium phosphate crystals in vitro and on aortic and kidney calcification in vivo.; Eur $J$ Clin Invest 1: 12-18.

Francis MD, Graham R, Russell G, et al. 1969; Diphosphonates inhibit formation of calcium phosphate crystals in vitro and pathological calcification in vivo. Science 165: 1264-1266.

Fujihara K, Kotaki M, Ramakrishna S. 2005; Guided bone regeneration membrane made of polycaprolactone/calcium carbonate composite nano-fibers. Biomaterials 26: 4139-4147.

Gao H, Gu Y, Ping Q. 2007; The implantable 5-fluorouracil-loaded poly(L-lactic acid) fibers prepared by wet-spinning from suspension. $J$ Control Release 118: 325-332.

Hae-Won K, Hae-Hyoung L, Knowles JC. 2006; Electrospinning biomedical nanocomposite fibers of hydroxyapatite/poly(lactic acid) for bone regeneration. J Biomed Mater Res A 79A: 643-649.
Hansen NM, Felix R, Bisaz S, Fleisch H. 1976; Aggregation of hydroxyapatite crystals, Biochim Biophys Acta Gen Subj 451: 549-559.

Horie D, Takahashi M, Aoki K, et al. 2003; Clodronate stimulates bone formation as well as inhibits bone resorption and increases bone mineral density in rats fed a low-calcium diet. $J$ Med Dent Sci 50: 121-132.

Huolman R, Ashammakhi N. 2007; New multifunctional anti-osteolytic releasing bioabsorbable implant. J Craniofac Surg 18: 295-301.

Hutmacher DW. 2000; Scaffolds in tissue engineering bone and cartilage. Biomaterials 21: 2529-2543.

Ito $\mathrm{Y}$, Hasuda $\mathrm{H}$, Kamitakahara $\mathrm{M}$, et al. 2005; A composite of hydroxyapatite with electrospun biodegradable nanofibers as a tissue engineering material. Soc Biotechnol Japan 100: 43-49.

Itoh $\mathrm{F}$, Aoyagi S, Furihata-Komatsu $\mathrm{H}$, et al. 2003; Clodronate stimulates osteoblast differentiation in ST2 and MC3T3-E1 cells and rat organ cultures. Eur $J$ Pharmacol 477: 9-16.

Jeppsson C, Astrand J, Tagil M, et al. 2003; A combination of bisphosphonate and BMP additives in impacted bone allografts. Acta Orthop Scand 74: 483-489.

Kikuchi M, Koyama Y, Yamada T, et al. 2004; Development of guided bone regeneration membrane composed of $\beta$ tricalcium phosphate and poly(L-lactideco-glycolide-co- $\varepsilon$-caprolactone) composites. Biomaterials 25: 5979-5986.

Kim HW, Knowles CJ, Kim HE. 2004; Effect of biphasic calcium phosphates on drug release and biological and mechanical properties of $\operatorname{poly}(\varepsilon$ caprolactone) composite membranes. $J$ Biomed Mater Res A 70A: 467-479.

Kim JH, Lee KH. 1998; Effect of PEG additive on membrane formation by phase inversion. $J$ Membr Sci 138: 153-163.

Koh YH, Bae CJ, Sun JJ, et al. 2006; Macrochanneled poly( $\varepsilon$-caprolactone)/ hydroxyapatite scaffold by combination of bi-axial machining and lamination. $J$ Mater Sci Mater Med, 17: 773-778.

Kokubo T, Kim HM, Kawashita M. 2003, Novel bioactive materials with different mechanical properties. Biomaterials 24: 2161-2175.

Lee KH, Baek DH, Ki CS, et al. 2007; Preparation and characterization of wet spun silk fibroin/poly(vinyl alcohol) blend filaments. Int $J$ Biol Macromol 41: $168-172$.

Leu C-T, Luegmayr E, Freedman LP, et al. 2006; Relative binding affinities of bisphosphonates for human bone and relationship to antiresorptive efficacy. Bone 38: 628-636.

Maretschek S, Greiner A, Kissel T. 2008; Electrospun biodegradable nanofiber nonwovens for controlled release of proteins. J Control Release 127: 180-187.

Martins A, Araujo J, Reis RL, et al. 2007; Electrospun nanostructured scaffolds for tissue engineering applications. Nanomedicine 2: 929-942.

Martins A, Chung S, Pedro AJ, et al. 2009a; Hierarchical starch-based fibrous scaffold for bone tissue engineering applications. $J$ Tissue Eng Regen Med 3: 37-42.

Martins A, Pinho ED, Faria S, et al. 2009b; Surface modification of electrospun polycaprolactone nanofiber meshes by plasma treatment to enhance biological performance. Small 5: 1195-1206.

McLeod K, Anderson GI, Dutta NK, et al. 2006; Adsorption of bisphosphonate onto hydroxyapatite using a novel coprecipitation technique for bone growth enhancement. $J$ Biomed Mater Res A 79A: 271-281.

Meyer JL, Nancollas GH. 1973; The influence of multidentate organic phosphonates on the crystal growth of hydroxyapatite. Calcif Tissue Int 13: 295-303.

Mulder M. 1996; Basic Principles of Membrane Technology. Kluwer Academic: Dordrecht.

Nafea EH, El-Massik MA, El-Khordagui LK, et al. 2008; Alendronate PLGA microspheres with high loading efficiency for dental applications. $J$ Microencaps 24: 525-538.

Nie H, Wang C-H. 2007; Fabrication and characterization of PLGA/HAp composite scaffolds for delivery of BMP-2 plasmid DNA. J Control Release 120: 111-121.

Ong H, Loo J, Boey F, et al. 2008; Exploiting the high-affinity phosphonate-hydroxyapatite nanoparticle interaction for delivery of radiation and drugs. J Nanopart Res 10: 141-150.

Ostović D, Stelmach C, Hulshizer B. 1993 Formation of a chromophoric complex between alendronate and copper(II) ions. Pharm Res 10: 470-472.

Patashnk S, Rabinovich L, Golomb G. 2009 Preparation and evaluation of chitosan microspheres containing bisphosphonates. J Drug Target 4: 371-380.

Perugini P, Genta I, Conti B, et al. 2001; Long-term release of clodronate from biodegradable microspheres. AAPS Pharm Sci Tech 2: 6-14.

Pinho ED, Martins A, Araújo JV, et al. 2009; Degradable particulate composite reinforced with nanofibres for biomedical applications. Acta Biomater 5: 1104-1114.

Piras AM, Chiellini F, Chiellini E, et al. 2008; New multicomponent bioerodible electrospun nanofibers for dual-controlled drug release. $J$ Bioact Compat Polym 23: 423-443.

Piras AM, Nikkola L, Chiellini F, et al. 2006; Development of diclofenac sodium releasing bio-erodible polymeric nanomats. J Nanosci Nanotechnol 6: 3310-3320.

Puppi D, Chiellini F, Piras AM, et al. 2010a; Polymeric materials for bone and cartilage repair. Prog Polym Sci 4: 403-440.

Puppi D, Detta N, Piras AM, et al. 2010b; Development of electrospun three-arm star poly( $\varepsilon$-caprolactone) meshes for tissue engineering application. Macromol Biosci [DOI: 10.1002/mabi.200900422].

Puppi D, Piras AM, Detta N, et al. 2010; Poly(lactic-co-glycolic acid) electrospun fibrous meshes for the controlled release of retinoic acid. Acta Biomater 6: 1258-1268.

Rai B, Oest ME, Dupont KM, et al. 2007; Combination of platelet-rich plasma with polycaprolactone-tricalcium phosphate scaffolds for segmental bone defect repair. $J$ Biomed Mater Res A 81A: 888-899. 
Reuvers AJ, Smolders CA. 1987; Formation of membranes by means of immersion precipitation: Part II. the mechanism of formation of membranes prepared from the system cellulose acetate-acetone-water. J Membr Sci 34: 67-86.

Rogers MJ, Gordon S, Benford HL, et al. 2000; Cellular and molecular mechanisms of action of bisphosphonates. Cancer 88: 2961-2978.

Samdancioglu S, Calis S, Sumnu M, et al. 2006; Formulation and in vitro evaluation of bisphosphonate loaded microspheres for implantation in osteolysis. Drug Dev Ind Pharm 32: 473-481.

Savarino L, Baldini N, Greco M, et al. 2007; The performance of poly- $\varepsilon$-caprolactone scaffolds in a rabbit femur model with and without autologous stromal cells and BMP4. Biomaterials 28: 3101-3109.

Schiller C, Epple M. 2003; Carbonated calcium phosphates are suitable $\mathrm{pH}$ stabilising fillers for biodegradable polyesters. Biomaterials 24: 2037-2043.

Selander KS, Monkkonen J, Karhukorpi EK, et al. 1996; Characteristics of clodronateinduced apoptosis in osteoclasts and macrophages. Mol Pharmacol 50: 1127-1138.

Seshima H, Yoshinari M, Takemoto S, et al. 2006; Control of bisphosphonate release using hydroxyapatite granules. $J$ Biomed Mater Res B 78B: 215-221.

Shin M, Yoshimoto H, Vacanti JP. 2004; In vivo bone tissue engineering using mesenchymal stem cells on a novel electrospun nanofibrous scaffold. Tissue Eng 10: 33.

Sill TJ, von Recum HA. 2008; Electrospinning: applications in drug delivery and tissue engineering. Biomaterials 29: 1989-2006.

Stitzel J, Liu J, Lee SJ, et al. 2006; Controlled fabrication of a biological vascular substitute. Biomaterials 27: 1088-1094.

Strathmann H, Kock K, Amar P, et al. 1975; The formation mechanism of asymmetric membranes. Desalination 16: 179-203.

Taylor MS, Daniels AU, Andriano KP, et al. 1994; Six bioabsorbable polymers: in vitro acute toxicity of accumulated degradation products. J Appl Biomater 5: 151-157.

Tengvall P, Skoglund B, Askendal A, et al. 2004; Surface immobilized bisphosphonate improves stainless-steel screw fixation in rats. Biomaterials 25: 2133-2138.

Tsay CS, Mchugh AJ. 1990; Mass transfer modeling of asymmetric membrane formation by phase inversion. $J$ Polym Sci B Polym Phys 28: 1327-1365.

Tsay CS, McHugh AJ. 1992; A rationale for structure formation during phase inversion. $J$ Polym Sci B Polym Phys 30: 309-313.

Tuzlakoglu K, Alves CM, Mano JF, et al. 2004; Production and characterization of chitosan fibers and 3D fiber mesh scaffolds for tissue engineering applications. Macromol Biosci 4: 811-819.

Tuzlakoglu K, Bolgen N, Salgado A, et al. 2005; Nano- and micro-fiber combined scaffolds: a new architecture for bone tissue engineering. J Mater Sci Mater Med 16: 1099-1104.

Tuzlakoglu K, Pashkuleva I, Rodrigues MT, et al. 2009; A new route to produce starchbased fiber mesh scaffolds by wet spinning and subsequent surface modification as a way to improve cell attachment and proliferation. J Biomed Mater Res A 92A: 369-377.

Tuzlakoglu K, Reis RL. 2008; Chitosanbased scaffolds in orthopaedic applications. In Natural-based Polymers for Biomedical Applications, Reis RL (ed). Woodhead: Cambridge; 357-373.

Um IC, Ki CS, Kweon H, et al. 2004; Wet spinning of silk polymer: II. Effect of drawing on the structural characteristics and properties of filament. Int $J$ Biol Macromol 34: 107-119.

Ural E, Kesenci K, Fambri L, et al. 2000; Poly(D,L-lactide $/ \varepsilon$-caprolactone)/

hydroxyapatite composites as bone filler: preparation and characterization. Biomaterials 21: 2147-2154.

Van Beek ER, Lowik CWGM, Papapoulos SE. 2002; Bisphosphonates suppress bone resorption by a direct effect on early osteoclast precursors without affecting the osteoclastogenic capacity of osteogenic cells: the role of protein geranylgeranylation in the action of nitrogencontaining bisphosphonates on osteoclast precursors. Bone 30: 64-70.

Weidenauer U, Bodmer D, Kissel T. 2003; Microencapsulation of hydrophilic drug substances using biodegradable polyesters. Part I: Evaluation of different techniques for the encapsulation of pamidronate di-sodium salt. $J$ Microencaps 20: 509-524.

Wienk IM, Boom RM, Beerlage MAM, et al. 1996; Recent advances in the formation of phase inversion membranes made from amorphous or semi-crystalline polymers. J Membr Sci 113: 361-371.

Williams JM, Adewunmi A, Schek RM, et al. 2005; Bone tissue engineering using polycaprolactone scaffolds fabricated via selective laser sintering. Biomaterials 26: 4817-4827.

Williamson MR, Coombes AGA. 2004; Gravity spinning of polycaprolactone fibres for applications in tissue engineering. Biomaterials 25: 459-465.

Xie J, Wang C-H. 2006; Electrospun microand nanofibers for sustained delivery of paclitaxel to treat C6 glioma in vitro. Pharm Res 23: 1817-1826.

Xu X, Chen X, Xu X, et al. 2006; BCNUloaded PEG-PLLA ultrafine fibers and their in vitro antitumor activity against glioma C6 cells. J Control Release 114: 307-316.

Yao J, Masuda H, Zhao C, et al. 2001; Artificial spinning and characterization of silk fiber from Bombyx mori silk fibroin in hexafluoroacetone hydrate. Macromolecules 35: 6-9.

Zhang X, Hua H, Shen X, et al. 2007; In vitro degradation and biocompatibility of poly(L-lactic acid)/chitosan fiber composites. Polymer 48: 1005-1011.

Zhang Y, Lim C, Ramakrishna S. 2005; Recent development of polymer nanofibers for biomedical and biotechnological applications. J Mater Sci Mater Med 16: 933-946.

Zheng-Ming H, Chuang-Long H, Aizhao Y, et al. 2006; Encapsulating drugs in biodegradable ultrafine fibers through coaxial electrospinning. $J$ Biomed Mater Res A 77A: 169-179. 